\title{
Mapping of Process Induced Dopant Redistributions by Electron Holography
}

\author{
Wolf-Dieter Rau, Alexander Orchowski \\ LEO Electron Microscopy, Carl-Zeiss-Str 56, D-73447 Oberkochen, Germany ${ }^{[3]}$
}

\begin{abstract}
We present dopant mapping examples in semiconductors by electron holography and outline their potential applications for experimental investigation of 2D dopant diffusion. Moreover, we address the technical challenges of the technique when applied to device structures with respect to quantification of the results in terms of the $2 \mathrm{D}$ pn junction potential.
\end{abstract}

Semiconductor devices consist of precisely placed foreign atoms to produce doping and composition variations, and hence a microscopically tailored electrostatic potential. Externally applied signals can then be used to modify this electrostatic "landscape", and hence control charge transport through the device. From electron holographic phase images one can directly map this electrostatic potential distribution after various stages of device fabrication, which is highly desirable in order to understand and control the fundamental solid state processes that govern device fabrication [1,2]. Of main interest here are the lateral redistributions of locally implanted doping profiles during typical anneal conditions.

An investigation of an anneal sequence in ultra shallow junction formation is shown in fig. 1. An ndoped Si substrate material has be locally implanted with B through a patterned mask. A series of samples have been prepared at different anneal stages. The lateral and vertical evolution of the highly $\mathrm{p}$ doped regions can be seen in the phase images of unmasked regions shown in fig1 (a-c), allowing for a qualitative evaluation of the respective junction formation process. Unfortunately, further quantification of these images in terms of local electrostatic potential distribution is not possible, due to charging of the nitride mask still residing on top of the Si substrate material, as can be seen from the non-flat phase distribution in the vacuum region. Removing any sources of charge build-up is therefore absolutely essential for quantitative interpretation.

An example for successful potential mapping by holography is shown in figs. $2 / 3$, where mask layers have been removed before sample preparation. Here the goal was the investigation of lateral outdiffusion from a $5 \mathrm{~nm}$ B doping peak located within a $20 \mathrm{~nm}$ thick $\mathrm{Si}_{0.8} \mathrm{Ge}_{0.2}$ layer after implanting an additional dose of $\mathrm{B}$ through a patterned surface mask. Fig. 2a shows a TEM image of an area around an opening in the mask in an implanted and annealed sample. Residual defects can be seen in the implanted region. The electron holographic phase image of the masked region on the right is shown in fig. $2 \mathrm{~b}$. The $\mathrm{SiGe}$ layer is visible as a bright stripe due to the higher mean inner potential of Ge. The dark stripes on either side of the layer are due to the electrostatic potential change in the depletion zones of the $n-p$ and $p-n$ junction on top and bottom of the SiGe layer, caused by outdiffusion of B from the SiGe region. The broadening does not cease abruptly at the edge of the mask opening, extending significantly beneath the mask. Fig. 3a shows the quantitative map of the potential distribution. A comparison with process simulation shown in fig $3 \mathrm{~b}$ can now be used to refine according parameters of dopant diffusion models [2].

\section{References}

[1] W.-D. Rau et al., Phys. Rev. Lett. 82, 19 (1999) 2614.

[2] A. Orchowski et al., accepted by Appl. Phys. Lett. (2001).

[3] This work has been performed at the IHP for Microelectronics, Frankfurt (Oder) and the University of Dresden, Germany. We gratefully acknowledge the support, valuable contributions and input of H. Rücker, P. Schwander, A. Ourmazd, H. Lichte and M. Lehmann 


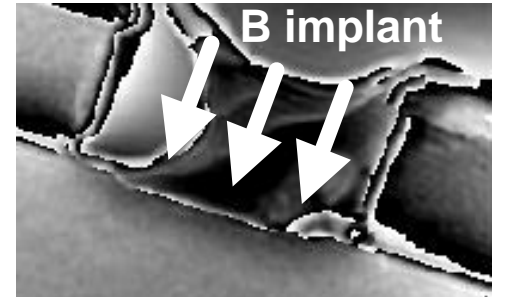

(a) $t=10 \mathrm{~s}$

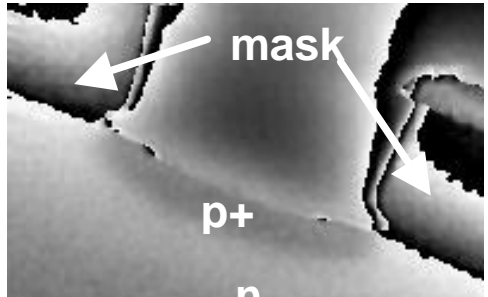

(b) $t=60 \mathrm{~s}$

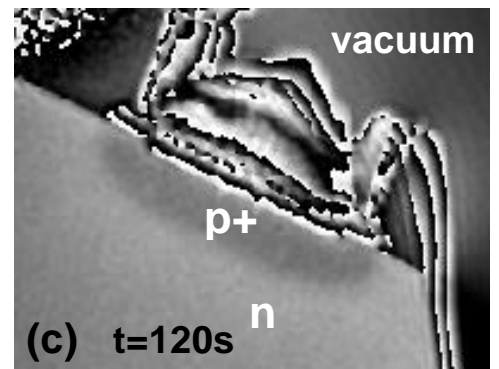

FIG. 1. Anneal sequence for Ultra Shallow Junction formation. The phase images reveal the vertical and lateral boron redistribution under the mask opening after a) $10 \mathrm{sec} \mathrm{b}) 60 \mathrm{sec}$ c) $120 \mathrm{sec}$ anneal.

FIG. 2. Mapping of lateral boron distribution at mask edge after boron contact implant in SiGe test structure. (a) Conventional TEM micrograph of implanted region. The mask was removed before sample preparation. (b) Phase image of the region boxed in (a). The SiGe layer appears as a bright stripe. The reduced phase in the dark regions reveals the electrostatic potential change due to B doping. $\mathrm{B}$ outdiffusion from the SiGe layer extends beneath he masked region.
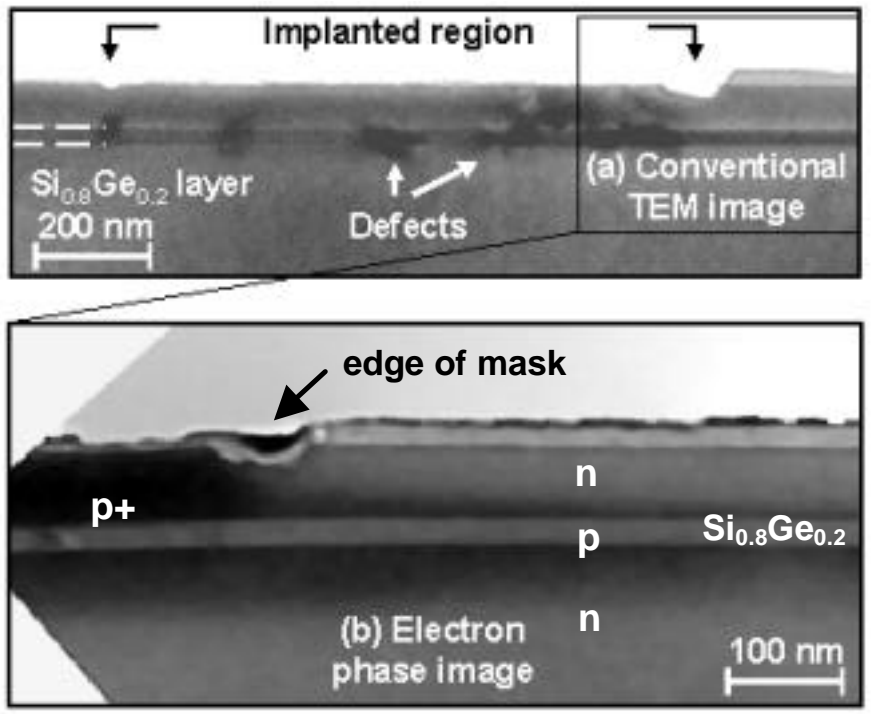

FIG. 3. (a) Experimental potential map derived from the phase image in fig. (b) Best matching process simulation of electrostatic potential distribution. The according dopant distribution can be used to extract diffusion model parameters for lateral B diffusion [2]
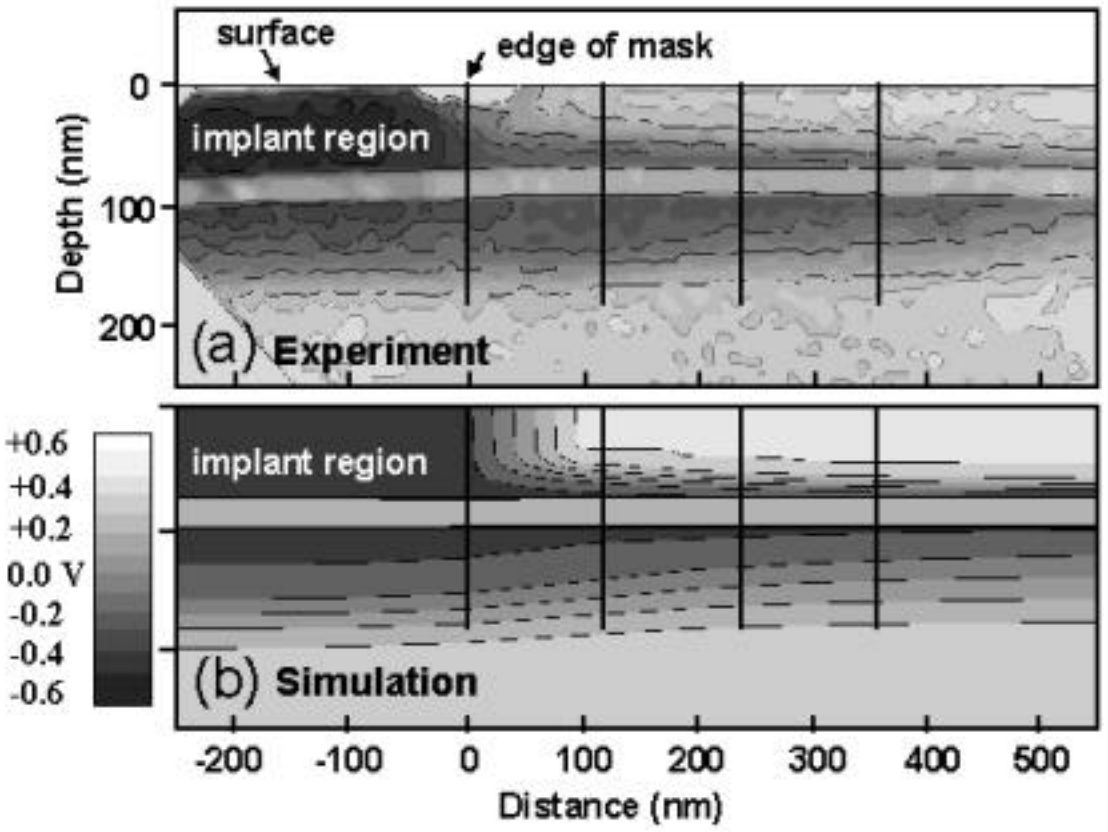